$\mathrm{p}=0.004)$, even after removing participants with cardiovascular disease (IGF-I: $\beta \quad-0.03 \mathrm{~ms} / \mathrm{ng} / \mathrm{ml} ; 95 \%$ CI -0.05 , $0.004 ; \mathrm{p}=0.021$; leptin: $\beta \quad-82.41 \mathrm{~ms} / \mathrm{ng} / \mathrm{ml} ; 95 \%$ CI 132.38, -31.92; $\mathrm{p}=0.001)$.

Conclusion Low levels of IGF-I and leptin (but not high leptin), representing insulin resistance, associate with prolonged QTc interval in older age. As QTc prolongation signals increased risk for sudden death even in apparently healthy people, public health efforts should be stepped up to combat the emergence of insulin resistance. Further research is needed to understand the potential mechanisms involved.

Conflict of Interest N/A

\section{FIVE-YEAR REAL WORLD DATA ON TRANSVENOUS LEAD EXTRACTION (TLE) IN A SINGLE LOW VOLUME CENTRE}

Kokhoon Tay, Rajesh Chelliah. University Hospitals of Leicester NHS Trust, Leicester, UK

\subsection{6/heartjnl-2021-BCS.93}

Introduction TLE procedure in low volume centres is associated with lower success rate, higher overall complication and mortality rates. We explored the indication of leads extraction, safety and re-implantation rate of cardiac implantable electronic device (CIED) post extraction in our low volume centre $[<30$ TLE procedures/ year as defined by the ELECTRa study, The European Lead Extraction Controlled study: a European Heart Rhythm Association (EHRA) Registry of Transvenous Lead Extraction Outcomes].

Method Retrospective 5-year analysis of all extraction of leads between 1st January 2016 to 31st December 2020 was retrieved from our electronic database system.

Results All TLE procedures were undertaken in cardiac catheter laboratory with 71 (78\%) male and 20 (22\%) female patients with mean (sd) age of 66 (14) years were included in the study. More than half $(n=50,55 \%)$ of the cohort had severely impaired left ventricular systolic function, 45 (50\%) patients were on oral anticoagulation therapy, 21 (23\%) patients with diabetes mellitus, $12(13 \%)$ with previous valvular heart surgery and 10 (11\%) patients had chronic kidney disease.Out of 115 leads extracted, 32 (28\%) were passive and $83(72 \%)$ were active leads. Almost half $(n=54,47 \%)$ of the leads extracted were ICD leads with one third of dual-coil leads. Maximum of four leads were extracted from one patient during same procedure. The overall mean (sd) dwelling time of leads is 6(7) years with the oldest lead had been in place for 29 years. Class 1 indication for extraction was predominantly pocket infection (47\%), followed by pocket erosion (24\%), CIED endocarditis (17\%) and lead failure (12\%). Mechanical extraction with laser technique (37\%) was the commonest method used followed by simple traction (24\%), locking stylets (20\%) and Evolution ${ }^{\circledR}$ mechanical dissecting sheaths (19\%). Complete procedural success was achieved in 95\% with one patient (aged 51) required emergency open chest extraction of active right atrial lead due to right atrial perforation and cardiac tamponade on table; the 14-year old lead was successfully retrieved without complication and he had an uneventful leadless pacemaker implantation.

The average length of hospitalization was 19 days for all patients. There was no directly attributed procedural mortality although a female patient (aged 50) with failed renal transplant on haemodialysis died of infected chest haematoma a week after successful extraction of her two 9-year old dualcoil ICD leads. Minor complication was extremely low with one patient requiring intercostal chest drain insertion due to pneumothorax from implantation of externalised pacemaker system after TLE procedure and another patient had localised superior vena cava dissection which healed. Approximately a quarter $(23 \%)$ of patients did not require CIED re-implantation, $11 \%$ of patients had re-implantation with leadless transcatheter pacemaker (Micra, Medtronic) and 6 (7\%) patients had subcutaneous ICD (Emblem, Boston Scientific) implantation after TLE procedures. There was no evidence of re-infection within 12 months in all patients.

Conclusion Infection remains the commonest important indication for TLE procedures and this study demonstrates clinical importance supporting the safety profile of competent operators undertaking TLE. The excellent outcome could be attributed in part to a single cardiologist operator undertaking the extraction procedures and therefore able to maintain competency achieving a minimum of 15 TLE procedures annually during the study. CIED infection carries important mortality and morbidity risks with significant implications when proceeding to system extraction. Leadless pacemaker or subcutaneous ICD implantation is a safe option in some subgroup of patients. Limitations: Although this is a retrospective study, it is likely similar good outcome could be conserved if our procedure volume rises exponentially enhancing operator skills.

Conflict of Interest None

\section{10-YEAR FOLLOW-UP OF ICD IMPLANTATION IN NON- ISCHAEMIC CARDIOMYOPATHY - AN INSIGHT FROM REAL WORLD PRACTICE IN A LARGE DISTRICT GENERAL HOSPITAL}

Heather Moore, Joanna Abramik, Gemina Doolub, Mark Dayer, Guy Furniss. Somerset NHS Foundation Trust, Taunton, UK

\subsection{6/heartjnl-2021-BCS.94}

Introduction The benefits of implantable cardioverter-defibrillators (ICDs) in ischaemic cardiomyopathy are well established. The DANISH study, a large randomized controlled trial of prophylactic ICD implantation, found no benefit in preventing all-cause mortality in patients with non-ischaemic cardiomyopathy (NICM). However, ESC guidelines recommend ICDs for patients with NICM and LVEF $\leq 35 \%$ who receive optimal medical therapy (IB recommendation). The 2014 NICE guidelines have similar endorsements but prior to that ICDs were not routinely recommended in NICM. We evaluated the longterm outcomes of patients who had ICDs implanted prior to 2014 in a NICM population at a large district general hospital.

Methods An ICD database of 454 patients at our district general hospital was retrospectively reviewed. We identified 70 patients who had ICD insertion for non-ischaemic cardiomyopathy between 2006 and 2014. Mean follow up time was 9 years 9 months (SD 23 months). 39 patients had an ICD inserted for primary prevention and 31 for secondary prevention. Data was collected from patients' electronic notes and analysed with relation to baseline demographics, mortality, complications and both appropriate and inappropriate therapy in those two subgroups. 